CARNETS OE Carnets de géographes

GÉOGRAPHES.

4 | 2012

Géographies critiques

\title{
A la recherche de la justice et de l'injustice en ville
}

Récit d'un terrain critique à Maputo

Karine Ginisty

\section{(2) OpenEdition}

Journals

Édition électronique

URL : http://journals.openedition.org/cdg/1003

DOI : $10.4000 /$ cdg. 1003

ISSN : 2107-7266

Éditeur

UMR 245 - CESSMA

Référence électronique

Karine Ginisty, «A la recherche de la justice et de l'injustice en ville », Carnets de géographes [En ligne], 4 | 2012, mis en ligne le 01 septembre 2012, consulté le 19 avril 2019. URL : http:// journals.openedition.org/cdg/1003; DOI : 10.4000/cdg.1003

Licence Creative Commons

La revue Carnets de géographes est mise à disposition selon les termes de la Licence Creative

Commons Attribution - Pas d'Utilisation Commerciale - Pas de Modification 4.0 International. 


\section{A la recherche de la justice et de l'injustice en ville récit d'un terrain critique à Maputo}

Karine Ginisty

ATER, Université Paris-Est Marne-la-Vallée Doctorante, Université Paris Ouest Nanterre la Défense k.ginisty@gmail.com

Tout se termine rua da justiça ${ }^{1}$, une rue parmi tant d'autres de Maputo. Sur ses trottoirs, les dalles de béton désagrégées, posées pour certaines il y a plus de 30 ans par le pouvoir colonial, tentent de survivre tant bien que mal au déracinement d'arbres en quête d'espace ; les voisins échangent les dernières salutations à la mode; les gardiens postés devant chaque maison s'endorment enfin sous les premiers rayons de soleil. Nous sommes dans les derniers jours d'août de l'année 2010 ; deux années viennent de s'écouler ; je ne reviendrai pas pour comprendre la place de l'espace dans la production de la justice et de l'injustice à Maputo. Désormais, je suis censée "savoir ». Ce "savoir » est pourtant loin de correspondre à un processus linéaire de recherche, où chacun des moments passés dans cette ville renverrait à un seul et même " objet " géographique. A mon arrivée à Maputo, je me suis intéressée à ce qu'Henri Lefebvre appelle la " production de l'espace ». Produit social, l'espace " [...] dénote et connote tous les espaces possibles, abstraits ou " réels " mentaux ou sociaux. " (Lefebvre, 1974 : 345). Ce qui fait espace interroge une multitude d'acteurs, de pratiques, de stratégies, de décisions, de rêves... En cherchant à saisir l'espace et son rôle dans la production de la justice et de l'injustice, mon terrain a peu à peu porté une démarche critique. Le terrain s'est tout d'abord présenté comme une pratique exploratoire de la ville. C'est en allant vers les citadins, les cadres techniques et les décideurs, en partageant des moments de vie jamais retranscris, que mon terrain a pris peu à peu forme ; il est devenu au fil des semaines, des mois, cet ensemble de moments privilégiés pour comprendre les réalités sociales qui $m^{\prime}$ entourent, dont je fais finalement partie intégrante. Si le terrain m'est avant tout apparu comme une expérience humaine, il a aussi contribué à la formation d'un regard critique sur les catégories dominantes utilisées pour penser et concevoir l'espace, qu'elles soient académiques ou ordinaires. En effet, à Maputo, le terrain permet la critique en offrant un espace de mise en visibilité des rapports de pouvoirs dans la production de l'espace. La critique est donc une démarche de questionnement qui porte le terrain jour après jour, et qui dans le même temps en est issue. Le récit qui suit tentera de restituer la construction d'une démarche critique par le terrain.

\footnotetext{
${ }^{1}$ Traduction française : rue de la justice ; nom de la rue où j'ai en partie résidé durant mon séjour à Maputo.
} 
Sur la route de l'aéroport, certaines scènes urbaines m'évoquent ma première traversée de la ville, en janvier 2007. Du suburbio ${ }^{2}$ je retiens un processus d'auto-construction hétéroclite, mêlant parpaings, tôles et roseaux, les défilés de bidons d'eau potable, jaunes et blancs, portés à bout de bras, placés sur la tête au petit matin, sans oublier les conversations animées, jamais très loin des petits étals commerçants qui vivotent jusqu'à la nuit tombée. Puis, en entrant dans la cidade ${ }^{3}$, le contraste interpelle toujours. Là, je reconnais l'héritage de l'impérialisme portugais, se démarquant par un urbanisme vertical et européen. Représentant le centre-ville actuel, la cidade domine Maputo; ses rues alignent la plupart des ressources urbaines - emplois, équipements, services, administrations. Rien ne semble avoir changé et pourtant rien n'est pareil. Cette grille de lecture duale de l'espace urbain, structurée autour du schème centre-périphérie ne m'apparaît plus opérante; elle est devenue une des représentations possibles de la ville, et non la seule. La distinction entre ces deux espaces repose sur l'espace conçu, " [...] celui des savoirs, des planificateurs, des urbanistes, des technocrates "découpeurs " ou "agenceurs [...] identifiant le vécu et le perçu au conçu » (Lefebvre, $1974: 48$ ). Dominée par les acteurs politiques et institutionnels, la dimension physique et matérielle de l'espace, propre à l'espace conçu, est un puissant instrument d'action sur la ville. A Maputo comme ailleurs, l'espace conçu tend pourtant à occulter les autres formes de production de l'espace. Si aujourd'hui je distingue ce qui relève du conçu, du perçu et du vécu dans la production de l'espace, je ne fus pas toujours en mesure de voir cette distinction et de la rendre visible par mon terrain. Je me souviens encore que, dans une ville méconnue, mes premiers pas de terrain ont reposé sur ce qui m'était donné à voir. En effet, pour saisir l'espace, j'ai tout d'abord été guidée par la dimension visible des services urbains - acteurs politiques et institutionnels, matérialité, débat public. Partie à la rencontre des acteurs impliqués dans la gestion et la fourniture des services urbains (bailleurs de fonds, municipalité, $A D M^{4}$ et le FIPAG), j'ai découvert une conception des services dominée par les questions distributives. La disponibilité, la densité, la répartition des infrastructures et des équipements au sein de la ville sont au cœur du débat public et des processus de décision, comme en attestent les projets menés pour atteindre les Objectifs du Millenium pour le Développement. Aussi, après seulement quelques semaines de terrain, formulais-je l'hypothèse d'un lien entre inégalités d'accès au réseau d'adduction d'eau potable et injustices sociales. L'orientation prise pour traiter mon objet de recherche me conduisit alors, du côté de l'action publique, à m'intéresser à la mise en place de traitements différenciés des territoires au sein de la ville, et à mobiliser les notions de territorialisation et de spatialisation des services urbains (Jaglin, 2005). Mon attention se focalisa particulièrement sur un projet de développement, le Maputo Water Supply Project, responsable de l'extension significative du réseau et de la reconnaissance des acteurs informels (appelés Petits Opérateurs Privés) dans la fourniture d'eau potable, ainsi que de la mise en œuvre d'un principe d'équité sociale dans la politique tarifaire. De ce point de vue, le rôle de l'espace semblait déterminant dans la production de la justice sociale en ville. Répondant à la rhétorique du développement, la réduction des inégalités intraurbaines entre la cidade et le suburbio figurait, plus généralement, à l'agenda du PRO

\footnotetext{
${ }^{2}$ Traduction française : suburbain (terminologie utilisée pour désigner les quartiers dits périphériques ou auto construits).

${ }^{3}$ Traduction française : ville (terminologie utilisée pour désigner le centre-ville de Maputo).

${ }^{4}$ Aguas de Moçambique est l'entreprise chargée d'exploiter et de gérer le réseau d'adduction d'eau potable, depuis 1999, dont la propriété demeure publique et à la charge du FIPAG (Fundo de Investimento e Património do Abastecimento de Agua).
} 
MAPUTO, premier Plan de Développement Municipal. Dans le même temps, j'entamais mes premiers entretiens qualitatifs avec les citadins dans les quartiers péricentraux du suburbio, Mafalala, Maxaquene, Polana Caniço et Urbanizaçao. La grille d'entretien retenue était centrée sur les modalités d'accès aux services. En s'intéressant aux stratégies développées pour pallier les déficiences, voire l'absence d'une fourniture de services, elle devait permettre de dégager des discours citadins sur le juste et l'injuste, en relation à l'action publique évoquée précédemment. Ces premiers contacts avec les citadins ont permis d'illustrer certaines réalités caractéristiques des villes du Sud : coûts des services plus élevés dans le suburbio que dans la cidade, prestations déficientes et risques sanitaires pour les citadins les plus précaires. Cependant, alors qu'était abordé le sujet des inégalités d'accès aux ressources urbaines, aucun des entretiens n'a permis de dégager de discours sur l'injustice. Le doute s'installait peu à peu... Les questions distributives me permettaient-elles réellement de comprendre la place de l'espace dans la production de la justice comme de l'injustice à Maputo ?

A la suite des émeutes urbaines du 5 février $2008^{5}$, où les citadins durent recourir à la violence, et la subir, pour faire entendre leurs revendications collectives auprès des pouvoirs publics, cette absence répétée de références à la notion d'injustice vint définitivement troubler la poursuite de mon terrain. Si ce silence pouvait être interprété comme la preuve de l'absence de sentiments d'injustices sociales, et donc de la réussite des politiques urbaines, il pouvait aussi être compris comme un indicateur de relations de domination et d'oppression (Young, 1990). Dans cette dernière hypothèse, le silence renvoie aux formes d'oppression dont fait l'objet un groupe ou une catégorie d'individus dans la société. Le silence est un produit social, renvoyant à ce qui ne peut être exprimé dans la sphère publique, politique, voire dans la sphère privée. Face au doute, je recentrais alors mes recherches sur les possibilités d'expression du juste et de l'injuste des citadins dans un contexte politique caractérisé par l'hégémonie du Frelimo ${ }^{6}$ sur la scène politique nationale et locale. En ce qui concerne le débat public, il ne semblait offrir que les registres discursifs des acteurs déjà reconnus dans les sphères publiques de discussion et de décision; les citadins apparaissaient exclus de la construction de l'action publique et de ses argumentaires ("lutte contre la pauvreté ", "développement»). De façon plus générale, en dehors des processus de décision, je ne trouvais pas de traces de mobilisations collectives structurées autour des services urbains et des inégalités afférentes. Je délaissais alors l'espace public, où les citadins demeurent invisibles, pour élaborer un guide d'entretien consacré aux expériences citadines des services urbains. J'entrepris de déconstruire avec les citadins leurs normes, valeurs et expériences du juste et de l'injuste. Ma démarche se heurta dans un premier temps à la méfiance, voire suscita de profondes inquiétudes. La justice se révèle être une notion critique. Dire l'injustice est en effet associé dans les représentations des citadins à la dissidence politique, à une remise en cause de l'Etat et plus spécifiquement des cadres du Parti dominant, le Frelimo. Il me fallut donc du temps pour mener un entretien qui se présentait comme un espace d'expression, ouvrant parfois même des débats collectifs au sein de la famille, entre amis, entre voisins. Il n'était pas seulement question d'aborder les représentations du juste et de l'injuste, mais aussi de comprendre les choix qui amenaient

\footnotetext{
${ }^{5} \mathrm{~A}$ l'annonce de l'augmentation des prix du transport collectif, les citadins prirent à partie les chauffeurs de chapas (mini-bus), bloquèrent et investirent les axes principaux de Maputo.

${ }^{6}$ Le FRELIMO (Frente de Libertação de Moçambique / Front de libération du Mozambique) est le parti politique au pouvoir depuis l'indépendance.
} 
les citadins à taire les injustices vécues. A l'image de l'entretien réalisé avec Borge, l'expérience des services urbains peut nourrir des sentiments d'injustice tus au quotidien (entretien, février 2009).

"Au sein de la ville, chaque maison devrait être équipée de l'eau potable, comme de l'électricité, parce que ça relève de ce qui prioritaire, mais ce n'est pas ce qui arrive. Et regarde les conséquences de tout ceci : tu te fais prendre à aller chercher de l'eau chez le voisin et le voisin reçoit une amende. [..] Il y a une grande injustice parce qu'ADM ne rend pas accessible l'eau pour les habitants, mais ceux qui ont l'eau et qui aident sont pénalisés. Et ils [les employés d'ADM] savent qu'ils n'ont pas les moyens de fournir l'eau, de créer des mécanismes pour faciliter l'accès à l'eau comme les bornes-fontaines. [..]

En vérité, jamais [je n'ai parlé au chef de Quarteirao et aux autres habitants de ces injustices]. Nous parlons entre nous, discutons entre amis, parce que ce sont des sujets... [..] il y a beaucoup d'injustices qui se produisent mais que faire ? A qui le crier ? Qui appeler au secours ? "

Du silence à la parole, l'idée de l'absence d'injustices sociales s'éloignait, et avec celle d'un lien significatif entre inégalités d'accès au réseau d'adduction d'eau potable et sentiments d'injustices sociales. Je délaissais la lecture des inégalités intra-urbaines faite par les " décideurs", politiques et institutionnels, qui ne me semblait plus déterminante dans la production du juste et de l'injuste en ville. Cette dernière repose sur une vision surplombante de l'espace, où le regard tend à se focaliser sur la question de la distance, notamment celle entre les différents points et lignes constitutifs des réseaux - conception géométrique et euclidienne de l'espace que l'on retrouve aussi en géographie. Je choisis de repenser mon terrain au regard de la pratique des services urbains, cherchant à rendre visible l'espace vécu des citadins, celui qui semble réduit à l'indicible. II me fallait comprendre ces représentations - espace perçu - où le paradigme de l'accessibilité n'est pas mobilisé dans la construction de discours sur le juste et l'injuste. II me restait à comprendre comment la mise en invisibilité de certaines pratiques citadines - par la négation politique ou le déplacement dans le registre de l'illégalité - participe à la formation d'injustices sociales à Maputo. Du doute à l'exploration de l'invisible, j'avais construit un terrain critique, m'amenant à explorer les raisons de la (non) traduction du sentiment personnel ou collectif de l'injustice dans les registres discursifs publics. Tout ne faisait que commencer.

\section{Références citées dans le texte}

Jaglin. S. (2005) Services d'eau en Afrique subsaharienne. La fragmentation urbaine en question, Paris, CNRS Editions, 244 p.

Lefebvre H. (1974) La production de l'espace, Paris, Anthropos, 485p.

Young I.M. (1990) Justice and the Politics of Difference, Princeton, New Jersey, Princeton University Press Books, 286 p. 